\title{
The Depression, Anxiety and Stress Scale (DASS-21) as a Screener for Depression in Substance Use Disorder Inpatients: A Pilot Study
}

\author{
Ilse N. Beaufort ${ }^{a, b}$ Gerdien H. De Weert-Van Oene ${ }^{a, b}$ Victor A.J. Buwalda ${ }^{a, b}$ \\ J. Rob J. de Leeuw ${ }^{c}$ Anna E. Goudriaan ${ }^{a}$ \\ ${ }^{a}$ Arkin Mental Health Amsterdam, Utrecht, The Netherlands; ${ }^{b}$ Jellinek Utrecht, Utrecht, The Netherlands; \\ 'Julius Center University Medical Center Utrecht, Utrecht, The Netherlands
}

\section{Keywords}

Validity - Diagnostics - Dual diagnosis - Substance use disorder - Depressive disorder · Screening instrument

\section{Abstract}

Depression is a common co-morbid disorder in substance use disorder (SUD) patients. Hence, valid instruments are needed to screen for depression in this subpopulation. In this study, the predictive validity of the Depression, Anxiety and Stress Scale (DASS-21) for the presence of a depressive disorder was investigated in SUD inpatients. Furthermore, differences between DASS-21 scores at intake and those recorded one week after inpatient detoxification were assessed in order to determine the measurement point of the assessment of the DASS-21 leading to the best predictive validity. The DASS-21 was administered to 47 patients at intake and shortly after inpatient detoxification. The results of the DASS-21 were compared to the Mini International Neuropsychiatric Interview (MINI), which served as the gold standard. Levels of sensitivity and specificity of $78-89 \%$ and $71-$ $76 \%$ were found for the DASS-21 assessed after detoxification, satisfactorily predicting depression as diagnosed with

\begin{tabular}{ll}
\hline KARGER & $\begin{array}{l}\text { C } 2017 \text { The Author(s) Karger } \\
\text { Published by S. Karger AG, Basel Open access }\end{array}$ \\
E-Mail karger@karger.com & This article is licensed under the Creative Commons Attribution- \\
www.karger.com/ear & $\begin{array}{l}\text { NonCommercial-NoDerivatives 4.0 International License (CC BY- } \\
\text { NC-ND) (http://www.karger.com/Services/OpenAccessLicense). } \\
\text { Usage and distribution for commercial purposes as well as any dis- } \\
\text { tribution of modified material requires written permission. }\end{array}$
\end{tabular}

the MINI. Total DASS-21 scores as well as the DASS subscale for depression were significantly reduced at the second measurement, compared to the DASS at intake. We conclude that the DASS-21 may be a suitable instrument to screen for depressive disorders in SUD patients when administered (shortly) after detoxification. Future research is needed to support this conclusion.

(c) 2017 The Author(s)

Published by S. Karger AG, Basel

\section{Introduction}

Converging results from international epidemiologic and clinical studies indicate that Substance Use Disorder (SUD) is often associated with depressive disorders and vice versa $[1,2]$. In the general population, $27 \%$ of individuals with a lifetime SUD are affected by a co-morbid lifetime major depression, whereas $41 \%$ of individuals with a lifetime major depressive episode also have a lifetime SUD [2]. Estimates of co-occurring depressive disorders in SUD treatment-seeking populations are even higher than those in the general population: $44 \%$ of patients included in SUD treatment facilities had a co-oc- 
curring major depression [3]. Nonetheless, reported prevalence of depression in SUD individuals seeking treatment varies strongly because of variability in study methods, such as differences in population sample, treatment setting, SUD under study, diagnostic instruments and difficulties in diagnosing [4]. Careful assessment of SUD patients is often hampered by overlapping symptoms and fluctuation of symptoms, since both active drug abuse and withdrawal from drugs can mimic symptoms of depression $[5,6]$. Additionally, psychiatric diagnostic procedures are time consuming. In a time where institutional budgets are limited, most SUD treatment facilities tend to give priority to their "core business" [7].

However, if not recognised early, SUD patients with co-morbid depressive disorder are more likely to have an unfavourable treatment outcome and tend to have a higher risk of relapse in substance abuse [8]. This emphasizes the need for reliable instruments to screen for depressive disorders in order to facilitate adequate treatment for comorbid depression in SUD patients.

An instrument that is often used to assess subjective depressive and anxiety complaints in patients is the Depression Anxiety Stress-Scale (DASS) developed by Lovibond and Lovibond [9]. The DASS has no direct implications for the allocation of patients to discrete diagnostic categories postulated in classification systems such as the diagnostic and statistical manual of mental disorders (DSM) and international classification of diseases and related health problems (ICD) [28], but it is predominantly aimed at assessing the perceived severity of symptoms related to depression, anxiety and stress [10].

The original 42-item DASS of Lovibond was modified into a shorter 21-item version [11]. Several studies are published on its reliability and validity worldwide, all showing the DASS-21 is a well-established instrument to measure symptoms of depression, anxiety and stress in both clinical and non-clinical samples of adults [11-16].

In the Netherlands, the DASS-21 is used in most SUD treatment facilities during intake in order to assess symptoms of depression, anxiety and stress in patients of 24 years and older. The questionnaire is used within the context of Routine Outcome Monitoring (ROM) to quantify and record the situation of a patient at a certain point in treatment, starting at intake [17]. In Dutch ROM, a score on the DASS-total of $\geq 60$ is considered an indication for further psychiatric assessment. It would be of great practical value if the DASS-21, being already used in daily practice, could serve as a screening instrument for depressive disorders (PTSD). Previously, Kok et al. [18] studied the DASS as a possible screener for post-traumatic stress disorder in SUD patients. These authors found the DASS to be a valid screening instrument for PTSD in SUD patients, when taken both at intake and 4 weeks after admission. However, to the best of our knowledge, no research has been done on the possible performance of the DASS- 21 as a screening instrument for depressive disorders in SUD patients.

The present study, therefore, tested the validity of the DASS-2 1 as a screening instrument for depression in SUD patients at 2 time points: when administered at intake as well as shortly after detoxification. We hypothesized the DASS- 21 would have a better predictive validity at the second time point (shortly after detoxification) than at the first time point (at treatment entry). Patients are not yet detoxified at intake, and acute alcohol and drug effects may affect the DASS scores. Moreover, patients may experience higher levels of negative emotional symptoms at treatment entry because of anxious anticipation for the admission and feelings of demoralization. The second time point is shortly after detoxification, and the effects of drugs and alcohol on the DASS scores are expected to be decreased. Hence, we were also interested in whether or not there were significant differences between the measured DASS scores at both time points. Studying the (possible) changes in DASS scores during this initial detoxification phase could lead us to the determination of the optimal time point of administering the DASS- 21.

\section{Methods}

\section{Participants}

This pilot study was conducted between April and July 2016. During 6 weeks, substance-dependent patients who entered the inpatient detoxification clinic of the SUD treatment facility in Utrecht in the Netherlands were eligible for participation. Exclusion criteria for this study were as follows: (1) patient admitted during an acute psychiatric crisis; (2) psychosis or delusion; (3) insufficient understanding of the Dutch language; (4) severe cognitive impairment. Patients' stay in this detoxification unit usually lasts for 10-15 days, and is followed by outpatient SUD treatment and, if necessary, treatment for co-morbid psychiatric disorder. Apart from being detoxified, with medical and psychological support, if necessary, patients are not being treated for their dependence or for their co-morbid disorders during this inpatient detoxification phase.

During the inclusion period, 98 patients were admitted to the detoxification clinic, of whom 51 patients participated in the study. In 47 patients, both the DASS-21 at measurement point 1 (intake) and 2 (one week after detoxification) were available for analyses (Fig. 1). No statistically significant differences were found between respondents and non-respondents regarding sociodemographic characteristics (Table 1), and with respect to mean scores on the DASS-21 at intake. However, non-participants more often reported the use of opioids or cocaine as their primary SUD problem. 
Fig. 1. Flowchart showing the participants

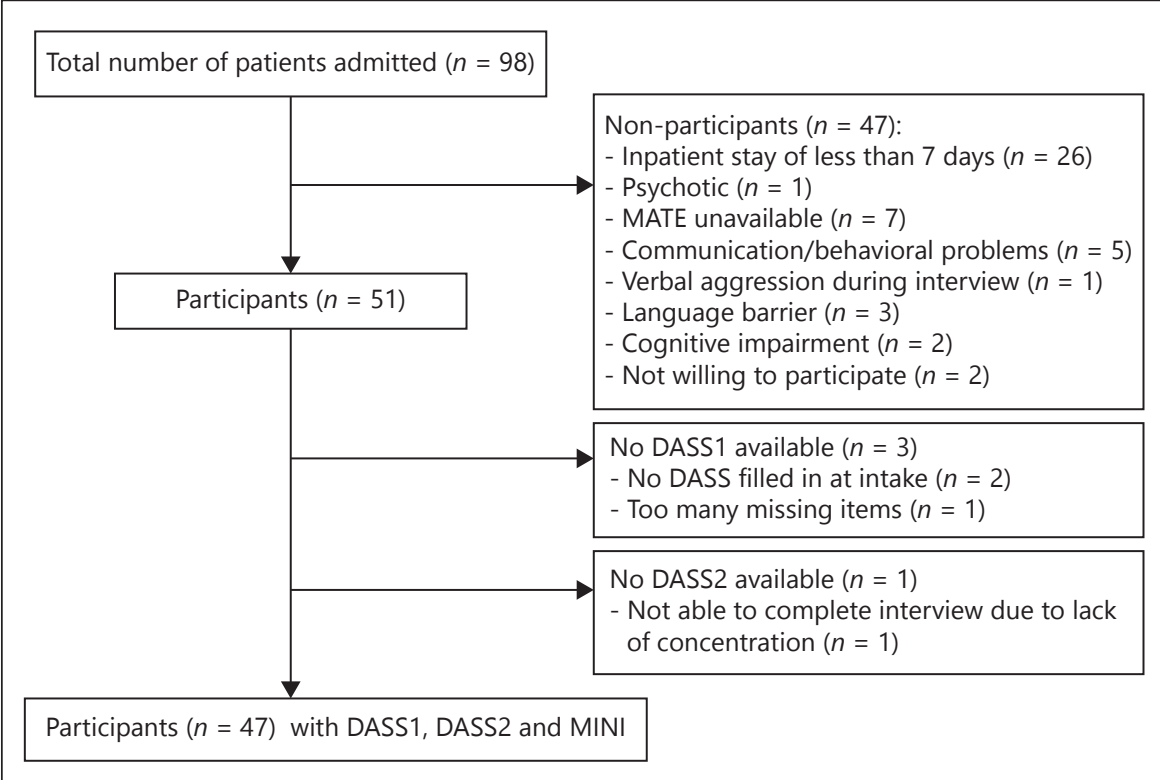
included in the study.

Table 1. Sociodemographic characteristics and psychoactive substance abuse of participants and nonparticipants

\begin{tabular}{|c|c|c|c|}
\hline & Participants $(n=47)$ & Nonparticipants $(n=47)$ & Statistics and significance \\
\hline \multicolumn{3}{|l|}{ Gender, \% } & \multirow[t]{3}{*}{$\mathrm{X}^{2}=0.224 ; p=0.636$} \\
\hline Male & 77 & 72 & \\
\hline Female & 23 & 28 & \\
\hline Age, mean (SD) & $43(13)$ & $40(11)$ & $\mathrm{T}=1.096 ; p=0.276$ \\
\hline \multicolumn{3}{|l|}{ Ethnicity, \% } & \multirow[t]{3}{*}{$\mathrm{X}^{2}=0.079 ; p=0.778$} \\
\hline Western & 85 & 83 & \\
\hline Non-Western & 15 & 17 & \\
\hline \multicolumn{3}{|l|}{ Marital status, $\%$} & \multirow[t]{4}{*}{$\mathrm{X}^{2}=2.797 ; p=0.247$} \\
\hline Married/registered partnership & 15 & 24 & \\
\hline Unmarried & 54 & 60 & \\
\hline Divorced/widowed & 31 & 16 & \\
\hline \multicolumn{3}{|l|}{ Daytime activity, $\%$} & \multirow[t]{3}{*}{$\mathrm{X}^{2}=0.311 ; p=0.577$} \\
\hline No regular daytime activity & 70 & 76 & \\
\hline Employed/studying & 30 & 24 & \\
\hline \multicolumn{3}{|l|}{ Primary problem substance, $\%$} & \multirow[t]{8}{*}{$\mathrm{X}^{2}=8.143 ; p=0.228$} \\
\hline Alcohol & 51 & 51 & \\
\hline Cannabis & 19 & 11 & \\
\hline Opiates & 2 & 11 & \\
\hline Cocaine/crack & 13 & 21 & \\
\hline Sedatives & 4 & 2 & \\
\hline GHB & 4 & 4 & \\
\hline Stimulants & 7 & 0 & \\
\hline \multicolumn{4}{|l|}{ Use of sedatives ${ }^{\mathrm{a}}, \%$} \\
\hline During detoxification & 87 & & \\
\hline Newly prescribed ${ }^{b}$ & 68 & & \\
\hline \multicolumn{4}{|l|}{ DASS scores at T1, mean (SD) } \\
\hline DASS total & $61.9(34.6)$ & $50.9(29.8)$ & $\mathrm{T}=-1.57 ; p=0.121$ \\
\hline DASS depression & $22.0(13.4)$ & $17.3(11.2)$ & $\mathrm{T}=-1.76 ; p=0.083$ \\
\hline
\end{tabular}

${ }^{a}$ Benzodiazepines, zopiclon or mirtazapine.

${ }^{\mathrm{b}}$ Prescribed during detoxification to reduce symptoms of withdrawal or sleeping problems. 


\section{Instruments}

Patients' sociodemographic background information was obtained from the patients' electronic record. Two measurement instruments were used to assess relevant patient characteristics: The Measurements in the Addictions for Triage and Evaluation, version 2.1 (MATE 2.1) and the Mini International Neuropsychiatric Interview (MINI).

The MATE 2.1 [19] is a mixed self-rating and expert-rating scale (semi-structured interview) to assess SUD and associated problems. The MATE 2.1 contains 10 modules: (1) the assessment of substance use, (2) indicators for psychiatric or medical consultation, (3) history of treatment for SUDs, (4) substance dependence and abuse, (5) physical complaints, (6) personality disorder, (7) activities and participation: care and support, (8) environmental factors influencing recovery, (Q1) craving and (Q2) depression, anxiety and stress. Module Q2 comprises the DASS-21. For this study, the relevant modules of the MATE 2.1 were (1) the assessment of substance use and (Q2) the DASS-21.

Module 1 assesses psychoactive substance use in the last 30 days and in the patient's entire lifetime. In this module, patients are asked which psychoactive substance(s) they use and the quantity consumed. In addition, patients are asked which substance is their primary-problem substance.

Module Q2, the DASS-21, is a self-report questionnaire consisting of 21 items, 7 items per subscale: depression, anxiety and stress. Patients are asked to score every item on a scale from 0 (did not apply to me at all) to 3 (applied to me very much). Sum scores are computed by adding up the scores on the items per (sub)scale and multiplying them by a factor 2 . Sum scores for the total DASStotal scale thus range between 0 and 120, and those for each of the subscales may range between 0 and 42 . Cut-off scores of 60 and 21 are used for the total DASS score and for the depression subscale respectively. These cut-off scores are derived from a set of severity ratings, proposed by Lovibond and Lovibond [20]. Scores $\geq 60$ (for DASS-total) and $\geq 21$ (for the depression subscale) are labeled as "high" or "severe". The reliability of the DASS-21 in this study population was $\alpha_{\text {dass_T1 }}=0.95\left(\alpha_{\text {dass_T1 }}=0.91\right.$ for depression subscale) and $\alpha_{\text {dass_T2 }}=0.92\left(\alpha_{\text {dass_T2 }}=0.86\right.$ for depression subscale).

The MINI [21, 22] is a structured diagnostic questionnaire for 23 Axis I mental disorders and 1 Axis II personality disorder (antisocial disorder). The scale consists of various modules, each focusing on different mental disorders. The questions are based on the DSM-IV (Diagnostic and Statistical Manual of Mental Disorders 4th Edition) and the ICD-10 (International Classification of Diseases 10th Edition), thereby allowing for a structured assessment of symptoms matching the most common mental disorders. The MINI distinguishes between current and lifetime episodes. In this study, all modules of the MINI were assessed.

\section{Procedure}

During intake, patients scored the DASS-21 (DASS_T1) as part of the standard intake procedure. After admission to the detoxification clinic, patients were asked to participate in this study and sign a written informed consent. This consent included permission to use data from the DASS-21 at intake, as well as sociodemographic data from their electronic patient record. The Medical Ethical Committee of the Academic Medical Center, University of Amsterdam, exempted this study from medical ethical review, due to the limited extra time needed by participants to fill out the DASS-21 at the second time point. The interview was performed by a trained interviewer and took place 8-10 days after the start of clinical detoxification when withdrawal symptoms were overcome. Patients filled out the DASS-21 a second time (DASS_T2) during the interview; additionally, the MINIinterview was completed, which served as the gold standard. Efforts were made to plan the second interview at least 2 days after possible use of sedatives was tapered off. In 4 out of 47 patients, this was not feasible and the interview took place on the last day of medication use $(n=3)$ or 1 day after the sedatives were stopped $(n=1)$. Three patients continued to use medication because of sleep-related problems.

\section{Data Analyses}

For sample representativeness, participants and non-participants were compared with regard to substance use and sociodemographic characteristics using $\mathrm{X}^{2}$ and $t$-tests. Sum scores for DASS scales were computed according to the Lovibond and Lovibond [9] severity ratings, resulting in "high" and "low" DASS scores. Subsequently, DASS scores of participants and non-participants were compared using $\mathrm{X}^{2}$-tests. Additionally, the percentages of patients with a DASS-total score of $\geq 60$, and of $\geq 21$ for the depression subscale respectively were assessed. Differences between DASS_T1, DASS_T2 and the depression subscale were tested using paired $t$ test analyses.

By using the MINI, sensitivity, specificity, and negative and positive predictive power were calculated for DASS_T1 and DASS_T2 separately. The discriminative accuracy of the DASS was evaluated by the receiver-operating characteristic (ROC) curve analysis. A ROC curve plots the sensitivity and specificity at each consecutive threshold value. The area under the ROC curve (AUC) is a suitable parameter to summarize the overall discriminative value of a screening instrument. A value of 0.70 or more can be interpreted as reasonable, 0.80 up to 0.90 as good and 0.90 or over as excellent $[23,24]$. Cut-off scores for sensitivity and specificity were obtained in 2 ways: (1) using the proposed cut-off scores in the MATE 2.1 (meaning cut-off values of 60 for total DASS score and 21 for depression subscale) and (2) determination of cut-off scores after conducting ROC analyses.

\section{Results}

Patients were predominantly male and of Western ethnic origin. Most of them were living alone. The majority of patients had no regular daytime activities. Fifty-one percent of patients indicated alcohol dependence as their primary problem (Table 1 ).

At treatment entry mean scores on the DASS_T1 total scale were $\mathrm{M}=61.9$ (SD 34.6), and those for the DASS_T1 depression subscale were $M=22.0$ (SD 13.4). At this time point, $53 \%$ of patients had a score above the threshold value for DASS-total and also 53\% for depression subscale, indicating severe complaints.

A significant difference was present between the DASS_T1 total score and the DASS_T2 total score $(p<$ 
Table 2. DASS scores of participants with and without a current depressive disorder

\begin{tabular}{|c|c|c|c|c|}
\hline DASS_T1 total score, mean (SD) & $61.9(34.6)$ & $68.7(17.1)$ & $60.3(37.6)$ & $\mathrm{T}=1.004 ; p=0.324$ \\
\hline DASS_T2 total score, mean (SD) & $33.7(23.9)$ & $52.0(24.2)$ & $29.3(22.0)$ & $\mathrm{T}=2.732 ; p=0.009$ \\
\hline T-test ${ }^{\bar{b}}$ & $\mathrm{~T}=5.783 ; p<0.001$ & $\mathrm{~T}=1.473 ; p=0.179$ & $\mathrm{~T}=5.693 ; p<0.001$ & \\
\hline DASS_T2 total & 15 & 22 & 13 & $\mathrm{X}^{2}=0.472 ; p=0.404$ \\
\hline DASS_T1 depression subscale, mean (SD) & $22.0(13.4)$ & $26.0(9.4)$ & $21.1(14.1)$ & $\mathrm{T}=0.988 ; p=0.328$ \\
\hline DASS_T2 depression subscale, mean (SD) & $11.2(10.2)$ & $19.6(9.8)$ & $9.3(9.4)$ & $\mathrm{T}=2.927 ; p=0.005$ \\
\hline T-test ${ }^{-1}$ & $\mathrm{~T}=5.329 ; p<0.001$ & $\mathrm{~T}=1.298 ; p=0.230$ & $\mathrm{~T}=5.344 ; p<0.001$ & \\
\hline \multicolumn{5}{|l|}{ Score above threshold ${ }^{¥ 2}$} \\
\hline DASS_T1 depression & 53 & 67 & 50 & $\mathrm{X}^{2}=0.387 ; p=0.407$ \\
\hline
\end{tabular}

0.001 , Cohen's $d=0.95)$. In addition, a significant reduction was observed in the depression subscale of DASS_T2 compared to DASS_T1 $(p<0.001$, Cohen's $d=0.91$; Table 2). At T2, mean scores on the DASS total scale were $\mathrm{M}=33.7$ (SD 23.9), and those for the depression subscale were $M=11.3$ (SD 10.2). At T2, $15 \%$ of patients had a score above the threshold value for DASStotal, and $19 \%$ for the depression subscale, indicating severe complaints. At T2, the MINI showed that $24 \%$ of patients were affected by any current depressive disorder. The lifetime prevalence of depressive disorder was $71 \%$.

Subsequently, mean DASS scores of participants with and without a current depressive disorder as assessed with the MINI were compared (Table 2). Participants with depressive disorder scored significantly higher on the DASS_T2 total score and the DASS_T2 depression subscale than participants without a depressive disorder; however, no differences were present between those patient categories on DASS_T1 scores.

Sensitivity, specificity, and positive and negative predictive power calculated for DASS_T1 and DASS T2 using different cut-off scores are shown in Table 3. No satisfactory results were obtained in predicting depressive disorder when using the cut-off values proposed by Lovibond and Lovibond [9]. To determine the cut-off values giving the best balance between sensitivity and specificity, ROC curves were computed.
This yielded statistically significant areas under the ROC-curves for DASS_T2 total score (AUC 0.795, 95\% CI $0.662-0.929 ; p=0.006)$ and DASS_T2 depression subscale (AUC $0.800,95 \%$ CI $0.664-0.936 ; p=0.006$ ) in predicting depression. According to the AUCs, the overall discriminative value of the DASS_T2 total score and DASS_T2 depression subscale can be interpreted as "good". For DASS_T2 total score, a cut-off score of 33 gave the best balance in sensitivity (89\%) and specificity $(71 \%)$ in predicting depressive disorders (Table 3). For DASS_T2 depression subscale, a score of 13 showed to be the best cut-off value, resulting in a reasonable sensitivity and specificity of $78 \%$ and $76 \%$ respectively.

Applying those new cut-off scores to the dataset yielded a percentage of $74 \%$ correctly identified cases for DASS_T2 total and 77\% for DASS_T2 depression scores. In addition, the differences between patients with and without a diagnosis of current depressive disorder were statistically significant, in contrast to those with the threshold values of 60 and 21 respectively.

\section{Discussion}

The first objective of this pilot study was to assess whether or not the DASS-21, when applied at intake in a SUD detoxification clinic and shortly after detoxifica- 
Table 3. Diagnostic value of the DASS_T1 and DASS_T2 at cut-off scores according to the MATE and alternative cut-off scores based on ROC-curves $(n=47)$

\begin{tabular}{|c|c|c|c|c|c|}
\hline & $\begin{array}{l}\text { Cut-off score } \\
\text { according to MATE }\end{array}$ & Sensitivity & Specificity & $\begin{array}{l}\text { Positive predictive } \\
\text { power }\end{array}$ & $\begin{array}{l}\text { Negative predictive } \\
\text { power }\end{array}$ \\
\hline DASS T1 total score & 60 & 0.67 & 0.50 & 0.24 & 0.86 \\
\hline DASS_T2 total score & 60 & 0.22 & 0.87 & 0.29 & 0.83 \\
\hline DASS_T2 depression subscale & 21 & 0.33 & 0.84 & 0.33 & 0.84 \\
\hline DASS_T1 total score & 57 & 0.78 & 0.47 & 0.26 & 0.90 \\
\hline DASS_T1 depression subscale & 23 & 0.67 & 0.53 & 0.24 & 0.86 \\
\hline DASS_T2 total score ${ }^{\mathrm{a}}$ & 33 & 0.89 & 0.71 & 0.42 & 0.96 \\
\hline DASS_T2 depression subscale ${ }^{a}$ & 13 & 0.78 & 0.76 & 0.44 & 0.94 \\
\hline
\end{tabular}

${ }^{a}$ Derived from statistically significant ROC-curves. Most favorable outcomes are printed in italics.

tion, can be used as screening instrument for depressive disorder in SUD patients. We found both a lack of sensitivity and specificity in predicting depression based on assessment at intake. Better results were obtained when taking the DASS shortly after detoxification, with the most favorable results achieved with the use of alternative cut-off values based on statistical analyses, for example, a score of 33 for DASS_T2-total and of 13 for DASS_T2depression -, lower than those proposed by Lovibond and Lovibond [9]. In addition, significantly higher scores on the DASS_T2 total score and DASS_T2 depression subscale were seen in participants who were diagnosed with a current depressive disorder on the MINI, compared to participants without a current depressive disorder.

The second objective was to evaluate whether there is a difference between DASS scores when assessed at intake and DASS scores assessed after clinical detoxification. We found significant reductions of DASS scores after detoxification compared to DASS scores at intake, both with respect to the DASS-total score and to the depression subscale.

The significant decrease of DASS_T2 total score and DASS_T2 depression subscale scores in comparison with the DASS_T1 scores suggests that patients experience more symptoms of depression at intake than shortly after (inpatient) detoxification. This supports our hypothesis that psychoactive substances and/or withdrawal symptoms may influence the results at treatment entry. In addition, anxious anticipation of the admission, insecurities concerning the future, and feelings of demoralization could be relevant explanations for the experienced negative emotions at this measurement time point. Since this study served as a first exploration of the subject, the possible effects of demoralization and of alcohol and drugs on feelings of depression, anxiety and stress were not included to be part of this study. This should be a topic of future research.

The prevalence of demoralization is high among SUD patients [25, 26]. Demoralization is defined as a psychological state characterized by both hopelessness and helplessness, and the inability to cope with stress that is expected to be handled by every individual [26, 27]. It is therefore possible that the symptoms experienced by the patient and assessed with the DASS- 21 at admission are more likely to reflect a temporary negative emotional state due to the factors mentioned before, rather than a depressive disorder. This is in line with the fact that the DASS is not intended to be used as a diagnostic instrument as the 3 subscales assess dimensional components of the anxiety and depressive disorders [28, 29].

This hypothesis is supported by the fact that no significant difference was found between the mean DASS T1 total score and mean DASS_T1 depression subscale scores for patients with and those without a depressive disorder. Therefore, we conclude that when taken at intake, the DASS-21 might not be able to discriminate between the overlapping symptoms of depression and strong emotions associated with substance use and the awaiting treatment. 
Application of the cut-off scores as proposed by Lovibond for DASS_T2 total score $(M=60)$ and DASS_ T2 depression subscale $(M=21)$, yielded reasonable levels of specificity, but low levels of sensitivity, thereby resulting in a considerable number of false-positives. However, in this study high sensitivity was favored over specificity in screening for depression. A low specificity for predicting depression using the DASS-21 results in many false-positives, which means a considerable number of MINI interviews will be administered unnecessarily. From an efficiency viewpoint, this is undesirable. Nonetheless, detecting as many depressive patients as possible has a strong clinical relevance. Depressive disorders in SUDs are associated with poorer treatment outcome, while this mental disorder can be adequately treated [8].

Cut-off scores obtained by ROC curves, being lower than those introduced by Lovibond and Lovibond [9], produced more satisfying results on both sensitivity and specificity. For predicting depression using the DASS-21 total score after detoxification, the best results were obtained with the highest sensitivity in this study (89\%), a negative predictive power of $96 \%$ and specificity of $71 \%$. Considering the better diagnostic value of the DASS-21 after detoxification and possible interfering factors when administering the DASS at intake, screening for depression shortly after detoxification is advisable. At this time point, influences of psychoactive substances or withdrawal symptoms are no longer present - or substantially less - and feelings of demoralization probably have diminished.

The proposed lower cut-off scores for the DASS-21 may be associated with (false-) positive MINI outcomes. In our study, the MINI was administered directly after detoxification, mainly for practical reasons. In most SUD treatment facilities, clinical detoxification lasts for 14 days followed by outpatient treatment. Patients with possible co-morbid depression need support and treatment, preferably as soon as possible in order to prevent treatment attrition and relapse in substance use. At the end of the detoxification phase, a treatment plan is set up, including referral to specialists for further assessment and diagnosis. The end of detoxification therefore seems to be a feasible point in time to screen for co-morbid disorders. However, there is some consensus in literature that the diagnosis of co-morbid psychiatric disorders is preferably done some 4 weeks after detoxification. On the other hand, Quello et al. [5] argued that the desired minimum amount of time before an evaluation of depression depends on the substance of abuse. SUD patients who are dependent on long-acting drugs may need a longer abstinence interval prior to diagnosis than patients using shorter-acting substances. In the present study, we studied a mixed population containing people dependent on various substances. The optimum time point for diagnosing depression in such a mixed SUD patient population should be the subject for further investigation.

Several strengths and limitations of this study need to be addressed. Sociodemographic characteristics and data of psychoactive substance use of participants were comparable to those of SUD patients in general according to the Dutch National Alcohol and Drugs Information System in 2014, indicating that participants - although limited in number - can be considered to be a fair representation of SUD patients in SUD treatment facilities in the Netherlands [30]. There was, however, an under-representation of opioid users among respondents compared to the general SUD population under treatment (2 vs. $14 \%$ ), which could be explained by a shorter average duration of stay for these patients in the clinical detoxification unit of only 4 days, due to a higher percentage of early attrition from treatment.

The results of this pilot study are based on data of a small sample of 47 patients. Because of this limited number, the number of patients with a current depression was relatively low, thereby lowering the statistical power. However, studies on the predictive validity of psychometric instruments are of fundamental scientific and clinical interest. Because of the favorable outcomes, this study could serve as a pilot study to confirm the utility of conducting larger studies on the predictive validity of the DASS-21 in SUD patients.

Furthermore, sedatives were prescribed in a vast majority of patients during the first week of clinical detoxification. These sedatives were already tapered off in most participants when the DASS-21 was administered for the second time, since efforts were made to schedule the second interview at least 2 days after the sedatives were tapered off. In 7 out of 47 patients this appeared not to be possible. This means that sedative effects might still have been present when the DASS-21 was filled-out by these patients. Sedatives are likely to mask symptoms of anxiety and stress but might do the same for symptoms of depression. The prescription of sedatives could have contributed to the lower scores on the DASS-21 total score and its subscales, in particular for patients with depressive disorder. However, this is not very likely, given the higher discriminative validity of the DASS-21 after detoxification, and the effective sensitivity and specificity for detecting 
current depression, compared with the MINI interview results.

In conclusion, SUD patients report significantly higher levels of symptoms of depression at intake compared to shortly after clinical detoxification. No significant differences were found between DASS-21 scores for patients with and without a depressive disorder at intake, whereas we did find significant differences after detoxification. It is therefore debatable whether at intake symptoms of demoralization, nervousness for admission and influences of psychoactive substance use are measured sooner than symptoms of depressive disorder. Based on the findings in this pilot study, we advise against the use of the DASS-21 at intake as a screening instrument for depressive disorders for SUD patients.
However, the DASS-21 seems to be a suitable instrument to screen for depressive disorder when scored after detoxification. The instrument may assist therapists to identify patients who are at high risk of being affected by a co-morbid clinical depression, in need of further diagnostic assessment. With regard to future research, a prospective study including a higher number of participants, preferably without the use of any sedatives or sedatives sufficiently tapered off, is needed to confirm these results.

\section{Disclosure Statement}

The authors report no conflicts of interest to disclose.

\section{References}

1 Regier DA, Farmer ME, Rae DS, Locke BZ, Keith SJ, Judd LL, Goodwin FK: Comorbidity of mental disorders with alcohol and other drug abuse. Results from the epidemiologic catchment area (ECA) study. JAMA 1990; 264:2511-2518.

-2 Kessler RC, McGonagle KA, Zhao S, Nelson $\mathrm{CB}$, Hughes M, Eshleman S, Wittchen HU, Kendler KS: Lifetime and 12-month prevalence of DSM-III-R psychiatric disorders in the United States. Results from the national comorbidity survey. Arch Gen Psychiatry 1994;51:8-19.

3 Bakken K, Landheim AS, Vaglum P: Axis I and II disorders as long-term predictors of mental distress: a six-year prospective followup of substance-dependent patients. BMC Psychiatry 2007;7:29.

4 European Monitoring Centre for Drugs and Drug Addiction: Co-morbid substance use and mental disorders in Europe: a review of the data, EMCDDA Papers, Publications Office of the European Union, Luxembourg, 2013.

5 Quello SB, Brady KT, Sonne SC: Mood disorders and substance use disorder: a complex comorbidity. Sci Pract Perspect 2005;3:1321.

6 Langas AM, Malt UF, Opjordsmoen S: Comorbid mental disorders in substance users from a single catchment area - a clinical study. BMC Psychiatry 2011;11:25.

7 Benjamin AB, Mossman D, Graves NS, Sanders RD: Tests of a symptom checklist to screen for comorbid psychiatric disorders in alcoholism. Compr Psychiatr 2006;47:227233.

8 Sheehan MF: Dual diagnosis. Psychiatry Quarterly 1993;64:107-134.

9 Lovibond SH, Lovibond PF: Manual for the Depression Anxiety Stress Scales. Sydney Psychology Foundation Australia 1995.
10 Lovibond P: Overview of the DASS and Its Uses. Retrieved from http://www2.psy.unsw. edu.au/dass/over.htm.

$\checkmark 11$ Antony MM, Bieling PJ, Cox BJ, Enns MW, Swinson RP: Psychometric properties of the 42 -item and 21-item versions of the depression anxiety stress scales in clinical groups and a community sample. Psychological Assessment 1998;10:176-181.

12 Sinclair SJ, Siefert CJ, Slavin-Mulford JM, Stein MB, Renna M, Blais MA: Psychometric evaluation and normative data for the depression, anxiety, and stress scales-21 (DASS-21) in a nonclinical sample of U.S. adults. Eval Health Prof 2012;35:259-279.

13 Henry JD, Crawford JR: The short-form version of the Depression anxiety stress scales (DASS-21): construct validity and normative data in a large non-clinical sample. Br J Clin Psychol 2015;44:227-239.

14 Bottesi G, Ghisi M, Altoè G, Conforti E, Melli G, Sica C: The Italian version of the Depression anxiety stress scales-21: factor structure and psychometric properties on community and clinical samples. Compr Psychiatry 2015; 60:170-181.

15 Tonsing KN: Psychometric properties and validation of Nepali version of the Depression Anxiety Stress Scales (DASS-21). Asian J Psychiatr 2014;8:63-66.

16 Vasconcelos-Raposo J, Fernandes HM, Teixeira CM: Factor structure and reliability of the depression, anxiety and stress scales in a large Portuguese community sample. Span J Psychol 2013;16:10.

17 Oudejans SC, Schippers GM, Merkx MJ, Schramade MH, Koeter MW, van den Brink W: Feasibility and validity of lowbudget telephonic follow-up interviews in routine outcome monitoring of substance abuse treatment. Addiction 2009;104:11381146.
18 Kok T, De Haan HA, Van Der Meer M, Najavits LM, De Jong CA: Screening of current post-traumatic stress disorder in patients with substance use disorder using the Depression, Anxiety, Stress scale (DASS-21): a reliable and convenient measure. Eur Addict Res 2015;21:71-77.

19 Schippers GM, Broekman TG, Buchholz A: MATE 2.1.: manual and protocol. Nijmegen Beta Boeken 2011.

20 Tran TD, Tran T, Fisher J: Validation of the depression anxiety stress scales (DASS) 21 as a screening instrument for depression and anxiety in a rural community-based cohort of northern Vietnamese women. BMC Psychiatry 2013;13:24.

21 Sheehan DV, Lecrubier Y, Sheehan KH, Amorim P, Janavs J, Weiller E, Hergueta T, Baker R, Dunbar GC: The Mini-International Neuropsychiatric Interview (M.I.N.I.): the development and validation of a structured diagnostic psychiatric interview for DSM-IV and ICD-10. J Clin Psychiatry 1998;59:2233.

22 Van Vliet IM, De Beurs E: The Mini International Neuropsychiatric Interview (MINI): a brief structured diagnostic psychiatric interview for DSM-IV and ICD-10 disorders (Het Mini Internationaal Neuropsychiatrisch Interview (mini): Een kort gestructureerd diagnostisch psychiatrisch interview voor dsm-iven icd-10-stoornissen). Tijdschrift voor Psychiatrie 2007;49:393-397.

23 Hanley JA, McNeil BJ: A method of comparing the areas under receiver operating characteristic curves derived from the same cases. Radiology 1983;148:839-843.

24 DeLong ER, Delong DM, Clarke-Pearson DL: Comparing the areas under two or more correlated receiver operating characteristic curves: a non-parametric approach. Biometrics $1988 ; 44: 817-845$. 
25 Tossani E, Ricci Garotti MG, Cosci F: The use of the diagnostic criteria for psychosomatic research in substance use disorder patients. Psychother Psychosom 2013;82:195-196.

-26 De Weert GH, Markus W, Kissane D, De Jong CAJ: Demoralization in patients with substance use disorder and co-occurring psychiatric disorders. J Dual Diagn 2017;13:136143.
27 Tecuta L, Tomba E, Grandi S, Fava GA: Demoralization: a systematicreview on itsclinicalcharacterization. Psychol Med 2015;45:673-691.

28 Psychology Foundation of Australia. (2011). Depression Anxiety Stress Scale - DASS. Retrieved from http://www2.psy.unsw.edu.au/ groups/dass//.

29 Osman A, Wong JL, Bagge CL, Freedenthal S, Gutierrez PM, Lozano G: The Depression
Anxiety Stress Scales-21 (DASS-21): further examination of dimensions, scale reliability, and correlates. J Clin Psychol 2012;68:13221338.

30 Wisselink DJ, Kuijpers WGT, Mol A: Core figures addiction treatment 2014 (Kerncijfers verslavingszorg 2014). Houten: Landelijk Alcohol en Drugs Informatie Systeem, 2015. 